\title{
Fuel gas blending benchmark for economic performance evaluation of advanced control and state estimation
}

\author{
N.L. Ricker ${ }^{\mathrm{a}}$, C.J. Muller ${ }^{\mathrm{b}, \mathrm{c}}$, I.K. Craig, ${ }^{\mathrm{c}, *}$ \\ ${ }^{a}$ Department of Chemical Engineering, University of Washington, Seattle, WA, USA. \\ ${ }^{b}$ Sasol Solvents RSA, Sasolburg, South Africa. \\ ${ }^{c}$ Department of Electrical, Electronic, and Computer Engineering, University of Pretoria, \\ Pretoria, South Africa.
}

\begin{abstract}
A simulation of a fuel gas blending process and its measurement system is proposed as a benchmark test case for advanced control and state estimation. The simulation represents an industrial facility and employs a well-established software environment. The objective is to maintain four controlled variables within specified bounds while minimizing an economic performance index. The controlled variables are the fuel gas pressure and three measures of gas quality. Six feed gas flow rates may be adjusted to achieve the objective. Each has a limited availability.

The benchmark consists of three reproducible scenarios, each a 46-hour period during which 23 discrete upsets occur and the feed gas compositions vary gradually with time. A benchmark multi-loop feedforward-feedback structure is described, tested, and compared to an estimate of optimal performance. The operating cost provided by the benchmark controller is from 1.19 to 1.71 times higher than the estimated minimum.

Readers are challenged to download the simulation model, benchmark controller and estimated optimal performance from the URL given in this paper, and to devise case studies of advanced state estimation and control strategies
\end{abstract}

\footnotetext{
*Corresponding author. Tel.: +27 124202172 .

Email addresses: ricker@u.washington.edu (N.L. Ricker), nelis.muller@sasol.com (C.J. Muller), icraig@postino.up.ac.za (I.K. Craig)
} 
to better the proposed benchmark controller.

Keywords: Real-time optimization, RTO, Advanced control, Ratio control, Nonlinear programming, Model predictive control, MPC, Nonlinear control

\section{Introduction}

An expectation of improved process economics often drives the installation of an advanced process control system. Such a system includes a regulatory component that attempts to maintain optimal process operation. This remains an active research area, as evidenced by many recent archival publications. For example, Darby et al. [1] review the state of the art in 2-level real-time optimization (RTO) systems, in which a detailed steady-state model determines the optimal operating point, which is used as setpoints for a lower-level advanced control system - typically a model predictive controller (MPC). Wurth et al. [2] describe a more general approach in which the optimization layer uses a model on a slow time scale, but not necessarily steady state. Jaschke and Skogestad [3] compare a self-optimizing control (SOC) in which the regulatory level inherently operates at a near-optimal condition to a strategy that formulates and tracks the necessary conditions for optimality (NCO tracking). Marchetti et al. [4] describe NCO tracking in which the gradients defining the necessary conditions are determined from real-time data rather than based on a model. Huang et al. [5] and Adetola and Guay [6] incorporate the economic objective directly in the nonlinear MPC. Shead et al. [7], Gopalakrishnan et al. [8], and Schei et al. [9] consider the related state estimation problem. Scattolini [10] reviews RTO in the context of a distributed rather than a centralized control structure. The review papers cited above should be consulted for a more comprehensive coverage of RTO methods.

There are also many recent application studies employing a variety of methods. For example, Ochoa et al. apply plant-wide one-level and two-level methods 
to a bioethanol production process, contrasting these to a more conventional decentralized RTO method [11]. Zavala et al. explicitly account for forecasting uncertainty in the optimal control of energy systems [12]. Porfirio and Odloak add an economic term to MPC for optimal operation of an industrial distillation system [13]. Chai et al. describe a hybrid system consisting of regulation, fault detection, and optimization components applied to an industrial roasting furnace [14]. Dewasme et al. apply extremum-seeking control to a fed-batch bioreactor, estimating gradients based on data rather than a mechanistic model [15]. Harinath et al. use nonlinear MPC for regulation and economic operation of a thermomechanical pulping process [16]. Chebre et al. [17] describe continuous and batch blending of liquid fuels to produce mixtures with prescribed properties while minimising the production cost. A key aspect is the use of an estimator to determine uncertain parameters.

Most such applications are complex or involve proprietary elements, which makes it difficult for other researchers to use them as benchmarks. The main contribution of the present paper is to provide a benchmark RTO application for use in research and education. The chosen process is the gas blending system considered in [18]. Blending is an important and generic industrial operation, which has, for example, motivated development of a patented RTO strategy $[19,20]$. It is conceptually simple and yet presents significant challenges. In comparison to the blending process described in [17], fuel gases are essentially ideal mixtures and their combustion properties are relatively easy to predict based on composition measurements. On the other hand, the gas blending system's mean residence time is relatively short, which suggests that rapid and frequent measurements are essential for effective feedback control.

Figure 1 is a flow diagram of a blending process within an integrated facility that steam-reforms natural gas to produce syngas [18]. The syngas is used on-site to make liquid fuels and waxes via the Fischer-Tropsch reaction. The continuous blending process combines six gases in a large header. Multiple 
Table 1: Controlled variable characteristics including measurement standard deviation, $\sigma$.

\begin{tabular}{|l|c|c|c|c|c|}
\hline Controlled Variable & Symbol & Units & Min & Max & $\sigma$ \\
\hline \hline Pressure & $\mathrm{P}$ & $\mathrm{kPa}$ & 2000 & 2200 & 5 \\
\hline Higher Heating Value & $\mathrm{HHV}$ & $\mathrm{MJ} / \mathrm{Nm}^{3}$ & 16.5 & 18 & 0.07 \\
\hline Wobbe Index & $\mathrm{WI}$ & $\mathrm{MJ} / \mathrm{Nm}^{3}$ & 25 & 27 & 0.07 \\
\hline Flame Speed Index & $\mathrm{FSI}$ & - & 39 & 46 & 0.07 \\
\hline
\end{tabular}

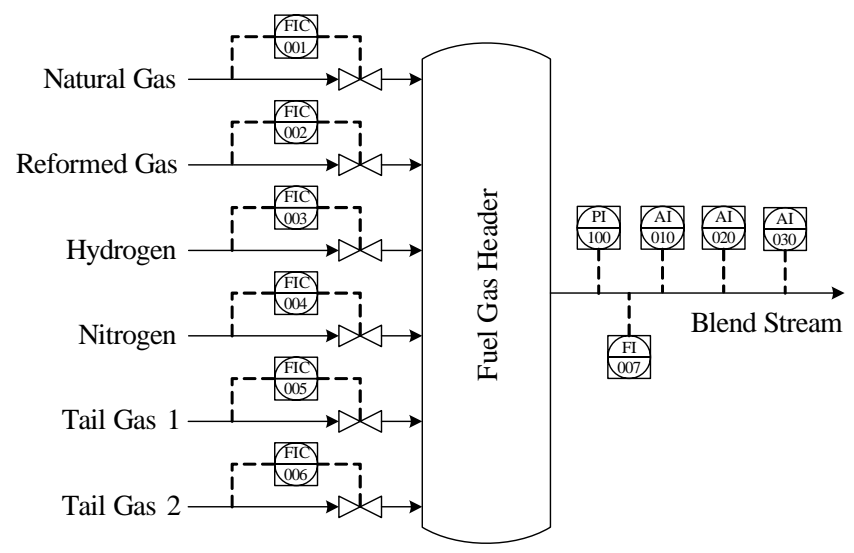

Figure 1: Fuel gas blending process flow diagram.

furnaces within the facility withdraw gas continuously, burning it to meet their heating needs.

The blending system must respond to significant changes in fuel gas demand and feed gas availability. It must also regulate the header pressure and meet specifications for high heating value (HHV), Wobbe index (WI), and Weaver's flame speed index (FSI). These depend on the fuel gas composition and must fall within the ranges shown in Table 1 . Table 2 summarizes the characteristics of each feed gas.

Another important objective is to minimize the blend cost. Three of the six feeds $\left(\mathrm{H}_{2}, \mathrm{TG}_{1}, \mathrm{TG}_{2}\right)$ are byproducts that would otherwise be flared, and thus are zero-cost. Two must be purchased $\left(\mathrm{NG}, \mathrm{N}_{2}\right)$ and one $(\mathrm{RG})$ is the synthesis 
Table 2: Feed stream characteristics.

\begin{tabular}{|c|c|c|c|}
\hline Feed & $\begin{array}{l}\text { Maximum } \\
\mathrm{kNm}^{3} / \mathrm{h}\end{array}$ & $\begin{array}{l}\text { Cost per } \\
\mathrm{kNm}^{3}\end{array}$ & Characteristics \\
\hline NG & 15 & 0.678 & $\begin{array}{l}\text { Natural gas. Always avail- } \\
\text { able. Composition changes step- } \\
\text { wise when facility switches gas } \\
\text { source. }\end{array}$ \\
\hline RG & 20 & 0.254 & $\begin{array}{l}\text { Reformed gas (syngas). Composi- } \\
\text { tion relatively constant but avail- } \\
\text { ability decreases } 50 \% \text { when one of } \\
\text { two syngas trains is out of service }\end{array}$ \\
\hline $\mathrm{H}_{2}$ & 5 & 0.0 & $\begin{array}{l}\text { Hydrogen byproduct. Constant } \\
\text { composition but availability can } \\
\text { drop to zero suddenly. }\end{array}$ \\
\hline $\mathrm{N}_{2}$ & 5 & 0.068 & $\begin{array}{l}\text { Nitrogen. Reliable supply and con- } \\
\text { stant composition. }\end{array}$ \\
\hline $\mathrm{TG}_{1}$ & 30 & 0.0 & $\begin{array}{l}\text { Tail gas } 1 \text {. Composition varies sig- } \\
\text { nificantly and availability can drop } \\
\text { to zero suddenly. }\end{array}$ \\
\hline $\mathrm{TG}_{2}$ & 30 & 0.0 & $\begin{array}{l}\text { Tail gas } 2 \text {. Characteristics similar } \\
\text { to } \mathrm{TG}_{1} \text {. }\end{array}$ \\
\hline
\end{tabular}


gas intended for use in Fischer-Tropsch synthesis. Thus, consumption of NG, $\mathrm{RG}$, and $\mathrm{N}_{2}$ should be the minimum meeting the fuel gas quality specifications and current demand.

Muller et al. [18] derived a mechanistic process model and validated it using data from the industrial facility. They also tested three versions of Model Predictive Control in a 6-hour simulated scenario in which demand, NG composition, and $\mathrm{TG}_{1}$ composition changed step-wise.

The present paper describes a more challenging scenario and embodies it in a MATLAB/Simulink simulation model that others may download. It includes an economic performance metric. For perspective, optimal performance is estimated and results are presented for a benchmark strategy employing timevarying ratio control with conventional feedforward-feedback header pressure control.

\section{Process description}

As in [18], the header states are the molar amounts of six chemical constituents: methane $\left(\mathrm{C}_{1}\right)$, heavier hydrocarbons $\left(\mathrm{C}_{2}^{+}\right), \mathrm{H}_{2}, \mathrm{~N}_{2}, \mathrm{CO}$, and $\mathrm{CO}_{2}$. The state equations are

$$
\frac{d N_{j}}{d t}=\sum_{i=1}^{6} y_{j i} F_{i}-y_{j} F
$$

where index $i=1$ to 6 refers to a feed stream, $j=1$ to 6 refers to a chemical constituent, $N_{j}$ is kmol of $j$ in the header, $y_{j}$ is the mole fraction of $j$ in the header, $y_{j i}$ is the mole fraction of $j$ in feed $i, F_{i}$ is the rate of feed $i, \mathrm{kmol} / \mathrm{h}$, and $F$ is the header discharge rate (demand), kmol/h. Equation 1 derives from the conservation of mass with assumptions of negligible chemical reactions and a well-mixed header. The header pressure is

$$
P=\frac{\sum_{j} N_{j} R T Z}{V}
$$


Table 3: Component characteristics.

\begin{tabular}{|c|c|c|c|c|}
\hline & HHV & M & A & s \\
\hline \hline $\mathrm{CH}_{4}$ & 37.78 & 16.04 & 9.55 & 148 \\
\hline $\mathrm{C} 2-\mathrm{C} 6$ & 126.5 & 58.12 & 31 & 514 \\
\hline $\mathrm{H}_{2}$ & 12.10 & 2.016 & 2.39 & 339 \\
\hline $\mathrm{N}_{2}$ & 0 & 28.02 & 0 & 0 \\
\hline $\mathrm{CO}$ & 11.97 & 28.01 & 2.39 & 61 \\
\hline $\mathrm{CO}_{2}$ & 0 & 44.01 & 0 & 0 \\
\hline
\end{tabular}

where $R$ is the universal gas constant, $T$ is the absolute temperature, $Z$ is the compressibility (assumed constant at $Z=1.06$ ), and $V$ is the header volume $\left(V=100 \mathrm{~m}^{3}\right)$. The temperature varies slowly in a diurnal cycle. Its impact on pressure is relatively small.

The remaining controlled variables depend on header gas composition according to

$$
\begin{gathered}
H H V=\sum_{j=1}^{6} y_{j} H H V_{j} \\
W I=\frac{H H V}{\sqrt{\rho}} \\
F S I=\frac{\sum_{j=1}^{6} y_{j} s_{i}}{\sum_{j=1}^{6} y_{j} A_{j}+5\left(y_{N_{2}}+y_{C O_{2}}\right)+1}
\end{gathered}
$$

where $s_{j}$ is the flame speed factor and $A_{j}$ is the molar stoichiometric air demand factor for component $j$. The specific gravity, $\rho$, is

$$
\rho=\frac{\sum_{j=1}^{6} y_{j} M_{j}}{M_{\text {air }}}
$$

where $M_{j}$ is the molar weight of component $j$ and $M_{\text {air }}=28.8$ is the molar weight of air. Table 3 lists the constants used in the above equations. 
The available manipulated variables are the six volumetric feed rates. These are expressed at standard conditions such that $1.0 \mathrm{kNm}^{3} / \mathrm{h}=44.64 \mathrm{kmol} / \mathrm{h}$. The nominal demand is $30 \mathrm{kNm}^{3} / \mathrm{h}$, so the mean header residence time is of order 4 minutes at the nominal pressure, $2100 \mathrm{kPa}$.

Downstream furnace operations set the demand. If it exceeds supply, the blending system operator must ask the furnace operators to reduce their consumption. Thus, the blending process control system cannot manipulate $F$, the total gas rate leaving the header.

In the industrial facility, gas demand depends on the HHV. For example, if furnaces are attempting to maintain constant temperature and the fuel gas HHV decreases, fuel demand increases. For simplicity, the benchmark simulation process omits this feedback mechanism.

\section{Measurements}

The measurement system is a key aspect of this application, and the present work models it more realistically than in [18]. The fundamental sampling period is 20 seconds, and all conventional measurements (feed and demand flow rates, header temperature and pressure) are available with negligible delay at this interval. The HHV and WI sensors also report every 20 seconds but include constant measurement delays of 1 and 3 intervals, respectively. The simulated measurements include random variability. The flow rate measurements also include systematic calibration error, which is an important issue in any blending system. Magnitudes of the random and systematic errors are representative of the industrial process.

A single mass spectrometer measures the compositions of the $\mathrm{NG}, \mathrm{RG}, \mathrm{TG}_{1}$, and $\mathrm{TG}_{2}$ feeds and the header composition sequentially. Each analysis requires two minutes, i.e., 10 minutes for the sequence of five measurements. The measurement sequence repeats from one cycle to the next but a measurement may 
be deemed unreliable, in which case the analyzer sets a status indicator. The model includes such events with probability 0.001 .

The fuel gas composition measured by the mass spectrometer determines the FSI. Consequently, a step-change in FSI would be detected with a delay ranging from 2 to 10 minutes, depending on when the change occurred in relation to the measurement sequence. The same would apply to detection of sudden feed gas composition changes.

The mass spectrometer readings are accurate for five of the six chemical constituents but the identity and relative amounts of the hydrocarbons comprising the $\mathrm{C}_{2}^{+}$fraction are unmeasured and time-varying. Consequently, the HHV and WI values one calculates using the spectrometer gas composition may disagree with the HHV and WI sensor readings (after adjusting for sampling delays). The latter are the quality measures to be compared to the specifications provided in Table 1. The spectrometer is the only source of FSI measurements and is its quality standard.

\section{The test scenario}

The gas blending process and measurement system described above may be downloaded as a MATLAB/Simulink model. An example control system is included (see Section 5). The reader is challenged to supply an alternative control system yielding better performance.

The performance metric is the average operating cost at the end of a 46-hour test scenario. Let $k$ denote a time instant $t_{k}=k \Delta t$ where the sampling interval is $\Delta t=20$ seconds, $k=0$ is the initial time, and $k=8280$ is the final time. The average cost at $t_{k}>0$ is

$$
\bar{C}\left(t_{k}\right)=\frac{\sum_{j=1}^{k} C\left(t_{j}\right)}{k} \forall k>0
$$

where $C\left(t_{k}\right)$ is the instantaneous cost at time $t_{k}$, defined as 


$$
C\left(t_{k}\right)=\frac{\sum_{i=1}^{6} c_{i} F_{i}\left(t_{k}\right)}{F\left(t_{k}\right)}+\delta_{k}
$$

where $c_{i}$ are the economic constants given in Table $2, F_{i}\left(t_{k}\right)$ and $F\left(t_{k}\right)$ are the actual feed and demand rates, respectively, and $\delta_{k}$ is a penalty function.

Unfortunately, it is impossible to quantify the economic cost of a constraint violation so the $\delta_{k}$ penalty is chosen as follows. Let the fuel gas specifications be

$$
C V^{L}-\epsilon_{k} \leq C V\left(t_{k}\right) \leq C V^{U}+\epsilon_{k}
$$

where $C V$ is the vector of four controlled variables from Table $1, C V^{L}$ and $C V^{U}$ are their specified lower and upper bounds, which are constant, and $\epsilon_{k} \geq 0$ is a vector quantifying the extent to which the four specifications have been violated at $t_{k}$. The penalty function employed here is

$$
\delta_{k}=0.02 \sum_{l=1}^{4}\left(\epsilon_{k, l} / \sigma_{l}\right)^{2}
$$

where $\sigma_{l}$ are the measurement uncertainties given in Table 1 . The rationale is that small violations could be due to measurement variability and would be ignored. The time-averaged cost is of order 0.1. Thus, the instantaneous cost of a violation $\epsilon_{k, l} \approx \sigma_{l}$ is about 0.02 . Infrequent violations of this magnitude have little impact on the time-averaged cost. On the other hand, persistent large violations would require a shut down or an expensive substitute fuel gas, and these receive a large penalty.

At each $t_{k} \geq 0$, the control system may adjust the setpoints of the six feed rate controllers shown in Figure 1. Table 4 lists all the signals the control system may exploit. These include the measurements described in Section 3, and the current availabilities of each feed. The simulation also provides $C\left(t_{k}\right)$, $\delta_{k}$, and $\bar{C}\left(t_{k}\right)$, but these signals are for evaluation only, not to inform controller decisions. 
Table 4: Simulation output signal characteristics.

\begin{tabular}{|l|l|l|l|}
\hline Symbol & Period $[\mathrm{s}]$ & Description & Units \\
\hline \hline$C V_{m}$ & 20 & $\begin{array}{l}\text { Four controlled variable mea- } \\
\text { surements. }\end{array}$ & see Table 1 \\
\hline$F_{m}$ & 20 & Measured demand. & $\mathrm{kNm}^{3} / \mathrm{h}$ \\
\hline$F_{i, \max }$ & 20 & Six feed rate availabilities. & $\mathrm{kNm}^{3} / \mathrm{h}$ \\
\hline$i_{s}$ & 120 & $\begin{array}{l}\text { Spectrometer status indicator } \\
\text { integer. }\end{array}$ & - \\
\hline$y_{i_{s}}$ & 120 & $\begin{array}{l}\text { Six measured mole fractions for } \\
\text { the stream indicated by } i_{s} .\end{array}$ & - \\
\hline$T_{m}$ & 20 & Header temperature. & $\mathrm{K}$ \\
\hline Cost & 20 & $\begin{array}{l}\text { Instantaneous and time- } \\
\text { averaged cost. }\end{array}$ & per $\mathrm{kNm}^{3}$ \\
\hline
\end{tabular}

Given the six manipulated variables and four controlled variables with defined ranges, one would expect at least two degrees of freedom to be available for cost minimization. Header pressure control requires the sum of the feed rates, $F_{i}$, to satisfy the demand, $F$. This can be done at any nominal header pressure. Thus, cost minimization requires at least one of the remaining three controlled variables and/or one or more feed rates to be at a bound. The minimum-cost condition will vary with the composition of the feeds, however, and it also depends strongly on feed availability. Thus, a strategy that attempts to maintain a single active constraint set will not be optimal and is likely to violate the controlled variable specifications.

The test scenario is a sequence of 23 unique events. These are typical upsets in the industrial facility but occur much more frequent (approximately every two hours) in order to limit a scenario to 46 hours. The events are sudden (step-like) changes in the following variables:

- Demand: nominal and $85 \%$ of nominal. 
- Reformed gas: nominal and 50\% availability.

- Hydrogen: nominal and zero availability.

- Tail gas 1: nominal and zero availability.

- Tail gas 2: nominal and zero availability.

There are 32 possible combinations. The 23 combinations employed in the test scenario were those remaining after elimination of simultaneous reductions in three or more availabilities. These would be rare and usually make it impossible to satisfy fuel gas demand. Given the header's open-loop response time of approximately 18 minutes (for composition changes), a control system should be able to respond completely to each event before the next occurs.

Throughout the 46 hours, the $\mathrm{RG}, \mathrm{TG}_{1}$, and $\mathrm{TG}_{2}$ compositions vary gradually within their typical ranges. The NG composition cycles between compositions representing the two different natural gas fields supplying the facility, and with a time-varying period on the order of four hours. The scenario also models a time-varying difference between HHV and WI measured by the sensors, and those calculated from the spectrometer measurements. This represents slow changes in the $\mathrm{C}_{2}^{+}$fraction as discussed in Section 3. The amplitude is approximately $\pm 0.8 \mathrm{MJ} / \mathrm{Nm}^{3}$ for both HHV and WI.

A candidate control system must react to the above events as if they were unexpected and of unknown duration. It must also infer all disturbances from the signals listed in Table 4 and the basic process description provided in Section 2. To emphasize the stochastic nature of the problem, the benchmark includes three different and reproducible realizations of the events and disturbances. A robust control system should provide comparable performance in all cases without case-specific parameter or tuning adjustments. 


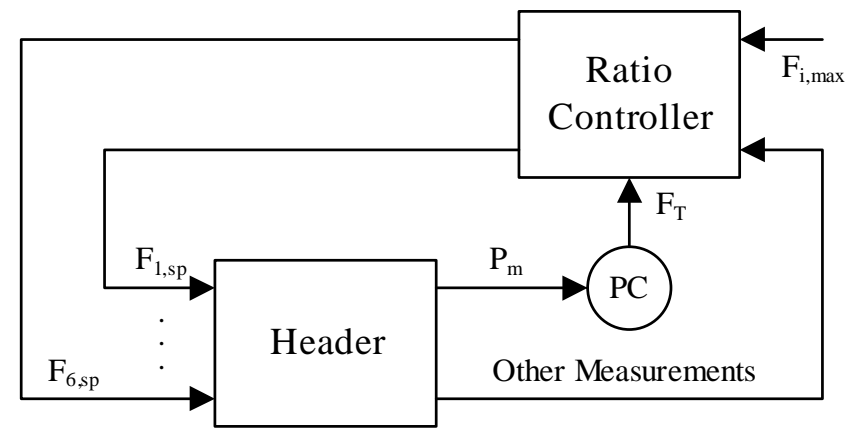

Figure 2: Benchmark controller block diagram.

\section{Benchmark: Pressure and feed-ratio control}

To provide a benchmark example the present work evaluates a combined pressure and feed-ratio-control strategy. Its block diagram appears in Figure 2. The pressure controller is feedforward plus single-input single-output, discretetime, proportional-integral feedback, specifically

$$
F_{T}(z)=F_{m}(z)+\left(2100-P_{m}(z)\right)\left(0.08+\frac{1.8 \Delta t}{z-1}\right)
$$

where $F_{m}$ is the measured demand, $P_{m}$ is the measured pressure, $2100 \mathrm{kPa}$ is the nominal pressure, and $\Delta t=20 / 3600 \mathrm{~h}$ is the control interval. The controller output, $F_{T}$, is the total feed flow rate to be supplied to the header, $\mathrm{kNm}^{3} / \mathrm{h}$. The feedback term corrects the nominal feedforward, which sets $F_{T}=F_{m}$. The above tuning constants yield good performance as long as feed availabilities allow the requested $F_{T}$ to be supplied.

The ratio controller multiplies $F_{T}$ by a vector of parameters, $r$, to determine setpoints of the six feed rate controllers (see Figure 1).

$$
F_{i}=r_{i} F_{T} \quad \forall i=1,6
$$

If feed compositions and availabilities were nearly constant, one could achieve adequate quality control using constant $r_{i}$ values. In the test scenario, however, 
large step-changes in availability make this untenable. Therefore, the example controller adjusts the ratios at each sampling instant, $t_{k}$, by solving the following nonlinear program

$$
\min _{r\left(t_{k}\right), \epsilon\left(t_{k}\right)} C\left(t_{k}\right)
$$

where $C\left(t_{k}\right)$ is the instantaneous cost defined by equation 8 . Minimization is subject to $\sum_{i=1}^{6} r_{i}\left(t_{k}\right)=1, \epsilon\left(t_{k}\right) \geq 0$, equation 12 , and the steady-state version of equation 1

$$
y_{j, s}=\sum_{i=1}^{6} r_{i} y_{j i, m}
$$

where $y_{j i, m}$ are the most recent feed composition measurements. In addition, the current availabilities (see Table 4) impose upper bounds on the ratios

$$
r_{i}\left(t_{k}\right) \leq \frac{F_{i, \max }\left(t_{k}\right)}{F_{T}\left(t_{k}\right)} \quad \forall i=1,6
$$

Also, the $y_{j, s}\left(t_{k}\right)$ values satisfying equation 14 are used in equations 3 to 5 to calculate corresponding quality measures $H H V_{s}, W I_{s}$, and $F S I_{s}$, which must satisfy three constraints of the form

$$
C V_{l}^{L}+\gamma_{l}-\epsilon_{l}\left(t_{k}\right) \leq C V_{s, l}\left(t_{k}\right)+b_{l}\left(t_{k}\right) \leq C V_{l}^{U}-\gamma_{l}+\epsilon_{l}\left(t_{k}\right), l=2,4
$$

where $C V_{s, l}\left(t_{k}\right)$ is the optimized quality measure, $C V_{l}^{L}$ and $C V_{l}^{U}$ are its specified bounds (Table 1 ), and $\epsilon_{l}\left(t_{k}\right)$ is the corresponding slack variable. Note that $\epsilon_{1}=0$ because the ratio controller is not designed to control header pressure. Thus, the NLP requires the optimization of nine decision variables at each $t_{k}$.

Equation 16 includes two features to make the controller more robust. The $\gamma_{l}$ are tunable safety margins. Their purpose is to reduce the likelihood of constraint violations. In all tests, $\gamma_{2}=\gamma_{3}=0.25, \gamma_{4}=0.50$. The $b_{l}\left(t_{k}\right)$ are 
feedback bias corrections. These compensate for the impact of unmeasured disturbances and sustained feed metering errors, and are calculated as follows:

$$
b_{l}\left(t_{k}\right)=b_{l}\left(t_{k-1}\right)+\alpha_{l}\left[C V_{s, l}\left(t_{k-d_{l}}\right)-C V_{m, l}\left(t_{k}\right)\right], l=2,4
$$

where $C V_{m, l}\left(t_{k}\right)$ is the measured quality, delay $d_{l}$ is chosen to compensate for the known measurement delay, and $0 \leq \alpha_{l} \leq 1$ is a constant noise filtering parameter. As in early forms of MPC, this bias correction forces a model prediction toward its measured value. In this case, however, the controller is using a steady-state (nonlinear) model rather than a dynamic model. In all tests, $\alpha_{l}=0.2, d_{2}=1, d_{3}=3$, and $d_{4}=6$. The initial conditions were $b_{l}\left(t_{0}\right)=0$. The $b_{l}$ values are updated each time a new measurement becomes available, i.e., every 20 seconds for HHV and WI, and every 10 minutes for FSI.

\section{Optimal performance estimate}

The true optimal time-averaged cost is useful for perspective when evaluating a proposed approach. If one considers the test scenario to be deterministic and assumes complete and accurate state measurements with no flow metering errors, a Mayer-type optimal control problem could be formulated, e.g.,

$$
\min _{u \subseteq U} \bar{C}\left(t_{f}\right)
$$

subject to

$$
\begin{gathered}
x(0)=x_{0} \\
\dot{x}=f(x, u, t)
\end{gathered}
$$

where $u$ represents the control actions, $U$ are the admissible control actions, $\bar{C}\left(t_{f}\right)$ is the time-averaged cost at the final time, $t_{f}, x$ is the state vector, $x_{0}$ is 
the initial condition, and $f(x, u, t)$ defines the state response to the controls and disturbances (see equation 1). If the control actions were piece-wise constant, varying every 20 seconds for 46 hours, $u$ would consist of 49680 variables to be optimized.

This problem could be solved in principle for a given deterministic scenario. However, the events should be considered unexpected and the disturbances are stochastic (Section 4). A controller designed to achieve the minimum cost for a given scenario is unlikely to be robust. A rigorous solution of the optimal control problem subject to uncertainty would be very difficult.

Therefore, optimal performance was estimated for each case rather than calculated rigorously. Two approaches were used. In the first, the ratio control strategy of Section 5 was simulated under the following ideal conditions

1. Accurate and continuous measurements of all gas compositions and fuel demand.

2. Accurate metering of the requested $F_{i}\left(t_{k}\right)$.

3. Noise-free sensor outputs with known sensor biases.

4. Safety margin $\gamma_{l}=0$ in equation 16 .

5. Small constraint violations (i.e., $\epsilon_{l} \leq \sigma_{l}$ ) were not penalized.

This minimizes the expected steady-state cost at each $t_{k}$.

The second approach also employed the above idealizations but considered the event-driven transients. Given the states at $t_{k}$, the process model (Section 2) was linearized as in [18] and used to predict CV values over a specified horizon. The optimization objective was to minimize the average cost over this horizon subject to the CV bounds. The resulting $F_{i}\left(t_{k}\right)$ values were implemented, and the optimization problem was reformulated at the next time instant, i.e., as in MPC. As both the steady-state and transient optimization approaches gave essentially the same results, details of the transient approach are omitted. This close agreement is probably an artifact of the header's fast mixing dynamics. 
Table 5: Benchmark results and estimated optimal cost

\begin{tabular}{|l|c|c|c|}
\hline Case & $\bar{C}$ & $\bar{\delta}$ & $\bar{C}_{\text {opt }}$ \\
\hline \hline 1 & 0.1331 & 0.0316 & 0.078 \\
\hline 2 & 0.1036 & 0.0048 & 0.087 \\
\hline 3 & 0.0944 & 0.0074 & 0.074 \\
\hline
\end{tabular}

The simpler steady-state formulation is unlikely to be optimal in general.

\section{Benchmark results}

Table 5 lists the time-averaged cost achieved by the benchmark ratio control strategy described in Section 5. For perspective, Table 5 includes the average penalty for constraint violations and the estimated optimal time-averaged cost. In the three cases studied, the benchmark strategy achieves a cost that is from 1.19 to 1.71 times higher than the estimated optimal cost.

Figures 3 to 5 show the Case 1 benchmark controller transients. The 23 discrete events appear as sudden changes in Figures 3 and 4. The observed drift between events is a combination of the transient response to control actions and the impact of stochastic disturbances.

Figure 3 shows the measured controlled variable and the fuel gas demand transients. The controlled-variable bounds appear as horizontal lines. The header pressure remains well within its bounds at all times. Thus, the benchmark strategy satisfies fuel gas demand under all conditions.

The benchmark strategy maintains WI near its lower bound. The HHV and FSI vary over their allowed ranges and exhibit lengthy periods at intermediate values. There are occasional HHV, WI, and FSI limit violations, which cause the instantaneous cost to spike. The FSI violations are the most serious, as they last at least 10 minutes (the FSI measurement interval) and increase the time-averaged cost about 30 percent in Case 1 . The chosen HHV and WI safety 
margins are such that their constraint violations are infrequent and contribute much less to the time-averaged cost.

In Figure 4, the blue transients are the controller feed adjustments and the feed availability variations are in green. The NG feed is essential to maintain the desired HHV. Thus, the benchmark strategy uses a non-zero NG feed at all times despite its high cost. It uses $\mathrm{TG}_{2}$ whenever possible, thereby reducing the NG rate. Most (but not all) events also employ large amounts of $\mathrm{TG}_{1}$ and $\mathrm{H}_{2}$. The $R G$ is zero unless at least two of the three zero-cost feeds $\left(\mathrm{H}_{2}, \mathrm{TG}_{1}, \mathrm{TG}_{2}\right)$ are unavailable, in which case the control system must employ RG in order to satisfy the fuel gas demand. (See for example the first event, which occurs after about 2 hours have elapsed.) The $\mathrm{N}_{2}$ rate is also zero during most events. Its main function is to reduce the FSI when the blend contains a relatively large $\mathrm{H}_{2}$ fraction.

The transients observed when estimating the optimal cost were qualitatively very similar to those shown in Figures 3 to 5 . (One may obtain these transients as explained in Section 9.) As would be expected, the optimal strategy reduces cost by holding one or more CVs at a bound, whereas the benchmark strategy employs safety margins. Consequently, the optimal strategy is able to increase the zero-cost feed utilization.

\section{Discussion and conclusions}

The blending process embodies the following challenges:

- Except for the pure feeds, gas composition must be inferred from infrequent mass-spectrometer measurements in combination with the more frequent but delayed HHV and WI sensor signals.

- Disturbances in feed mixture concentrations cause the impact of a given feed rate adjustment to vary with time. Feedback compensation is essential. If the feedback system employs a linear time-invariant (LTI) model, 

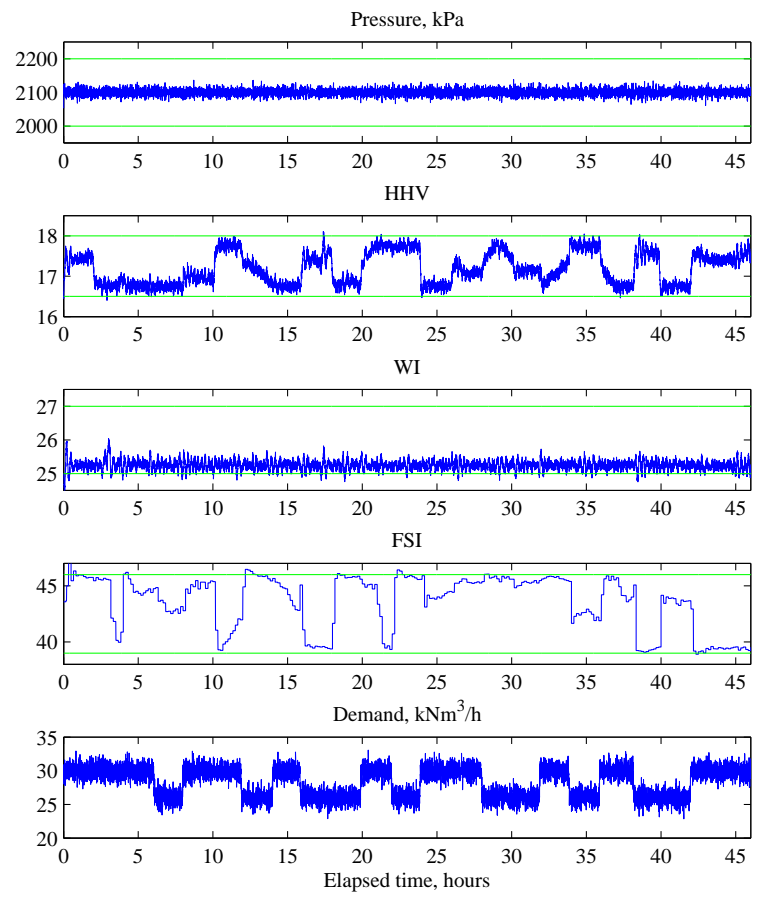

Figure 3: Controlled variables and fuel gas demand, Case 1. 

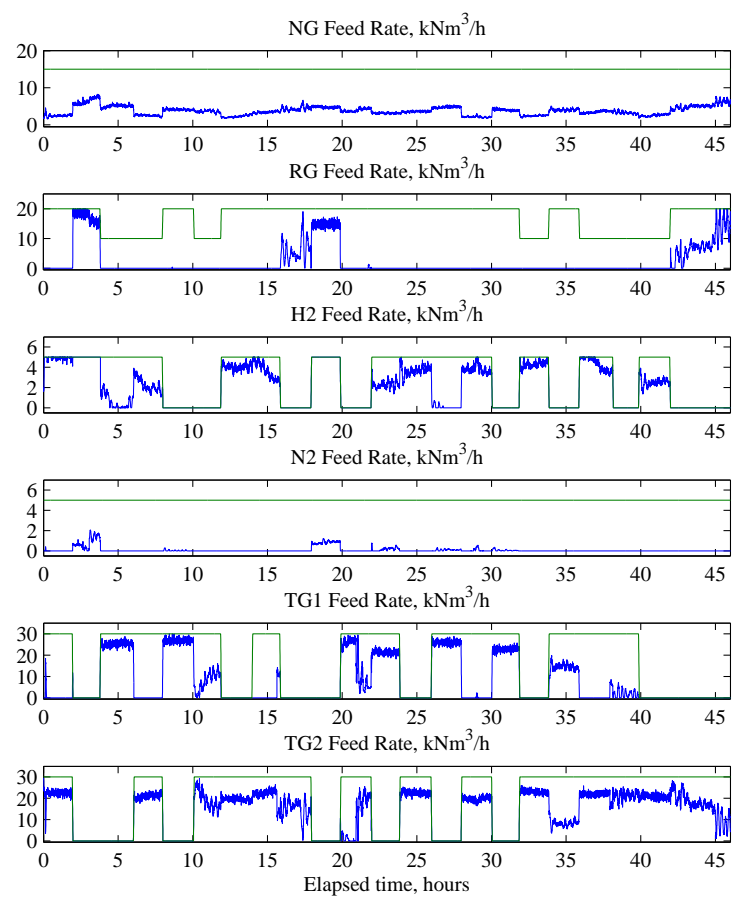

Figure 4: Feed rate adjustments and upper bounds, Case 1. 

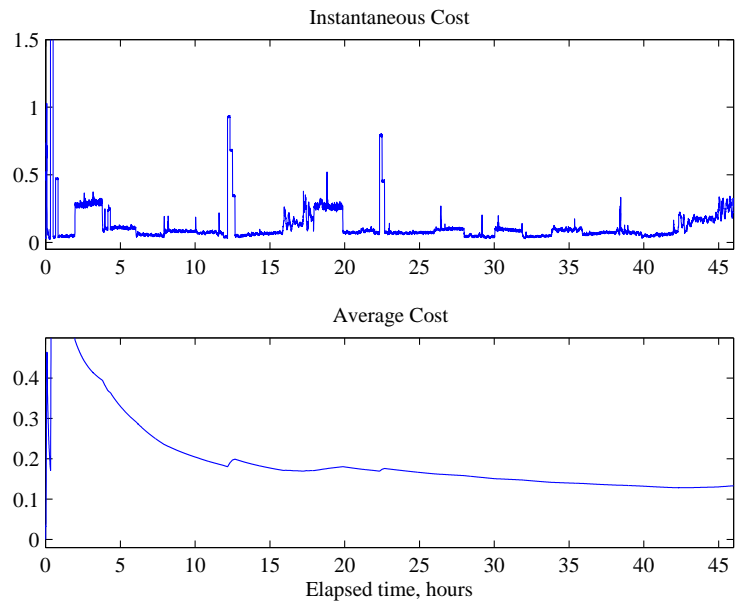

Figure 5: Instantaneous and time-averaged cost, Case 1.

feed composition variations would cause significant model error, e.g., an incorrect sign in one or more steady-state gains.

- Systematic metering errors also require feedback compensation.

- The sequential cyclic measurements of feed composition and header gas composition cause an apparent time-varying delay in the response of the gas quality measurements to feed rate adjustments.

The benchmark controller is a conventional multi-loop feedforward/feedback system. It solves a steady-state optimization problem at each sampling instant, but this is common practice in RTO. Moreover, the NLP dimension is low (nine decision variables) and its solution could be calculated rapidly using, e.g., typical spreadsheet software. The NLP component provides near-optimal handling of sudden changes in feed availability. It also compensates for sustained disturbances provided that these are estimated accurately via the feedback bias correction. 
A potential weakness is its focus on a future steady-state, which might allow an unfavorable transient state. This was not an issue in the benchmark scenarios because the mixing dynamics are fast and open-loop stable. Also, although the WI and FSI are nonlinear functions of gas composition, in the benchmark's composition range the responses to step changes in feed rates are unimodal. If this had not been the case, large WI and FSI constraint violations could have occurred during event transients. Other blending applications might not exhibit these favorable properties.

The benchmark pressure control loop was robust under all conditions. The benchmark ratio controller was sensitive to its adjustable parameters, but the above results were obtained through a small number of tuning trials, as is typical in practice. Constraint safety margin selection $\left(\gamma_{l}\right)$ was the most critical; it is likely that a better set could be found. In case 1, for example, the penalty for constraint violations is nearly 25 percent of the total cost. An increase in one or more $\gamma_{l}$ values might decrease the penalty significantly with a relatively small increase in feed costs. Adjustments in the feedback noise filter parameter, $\alpha_{l}$, had little effect near the recommended value, but performance deteriorated significantly at the extremes of $\alpha_{l} \approx 0$ (no feedback) and $\alpha_{l} \approx 1$ (no filtering).

As noted previously, in two of the three cases the benchmark comes within $25 \%$ the estimated optimal performance. It may be difficult for a more advanced and robust RTO strategy to achieve significantly better performance. The patented approach [19] employs nonlinear MPC and an extended Kalman filter for state estimation. Muller et al. [18] used a similar MPC-based approach but the measurement system model differed significantly from the more realistic benchmark model. Consequently, their controller could not achieve acceptable benchmark performance. However, as noted in Section 6, an MPC operating under ideal conditions (full state measurement, etc.) provided an estimate of optimal operation. Thus, it should be possible to improve the MPC of Muller et al. [18] by altering its state estimation strategy. Also, many other advanced 
RTO approaches could be applied to the benchmark. In order to encourage other researchers to contribute in this respect, the present authors will not publish future studies involving the fuel gas blending benchmark.

\section{Available materials}

The following may be downloaded from http://faculty.washington.edu/ ricker/Blending_Benchmark/Benchmark.htm:

- A MATLAB/Simulink model of the blending system process with the benchmark control strategy. The model was developed using MATLAB release R2011a. It is unlikely to function under earlier releases. Solution of the benchmark strategy's NLP requires the MATLAB Optimization Toolbox.

- Time series data for the three cases under the benchmark control strategy and the estimated optimal control.

[1] M.L. Darby, M. Nikolaou, J. Jones, and D. Nicholson. RTO: An overview and assessment of current practice. J. Process Control, 21(6), pp. 874-884, 2011.

[2] L. Wurth, R. Hannemann, and W. Marquardt. A two-layer architecture for economically optimal process control and operation. J. Process Control, 21(3), pp. 311-321, 2011.

[3] J. Jaschke and S. Skogestad. NCO tracking and self-optimizing control in the context of real-time optimization. J. Process Control, in press, 2011.

[4] A. Marchetti, B. Chacuat, and D. Bonvin. A dual modifier-adaptation approach for real-time optimization. J. Process Control, 20(9), pp. 10271037, 2010. 
[5] R. Huang, E. Harinath, and L.T. Biegler. Lyapunov stability of economically oriented NMPC for cyclic processes. J. Process Control, 21(4), pp. 501-509, 2011.

[6] V. Adetola and M. Guay. Integration of real-time optimization and model predictive control. J. Process Control, 20(2), pp. 125-133, 2010.

[7] L.R.E. Shead, K.R. Muske, and J.A. Rossiter. Conditions for which linear MPC converges to the correct target. J. Process Control, 20(10), pp. 1243-1251, 2010.

[8] A. Gopalakrishnan, N.S. Kaisare, S. Narasimhan. Incorporating delayed and infrequent measurements in extended Kalman Filter based nonlinear state estimation. J. Process Control, 21, pp. 119-129, 2011.

[9] T.S. Schei. On-line estimation for process control and optimization applications. J. Process Control, 18(9), pp. 821-828, 2008.

[10] R. Scattolini. Architectures for distributed predictive control - a review. J. Process Control, 19(5), pp. 723-731, 2009.

[11] S. Ochoa, G. Wozny, and J.U. Repke. Plantwide optimizing control of a continuous bioethanol production process. J. Process Control, 20(10), pp. 983-998, 2010.

[12] V.M. Zavala, E.M. Constantinescu, T. Krause, and M. Anitescu. On-line economic optimization of energy systems using weather forecast information. J. Process Control, 19(2009), pp. 1725-1736, 2009.

[13] C.R. Porfirio and D. Odloak. Optimizing model predictive control of an industrial distillation column. Control Engineering Practice, 19(10), pp. 1137-1146, 2011. 
[14] T. Chai, J. Ding, and F. Wu. Hybrid intelligent control for optimal operation of shaft furnace roasting process. Control Engineering Practice, 18(7), pp. 264-275, 2011.

[15] L. Dewasme, B. Srinivasan, M. Perrier, and A. Vande Wouwer. Extremumseeking algorithm design for fed-batch cultures of microorganisms with overflow metabolism. J. Process Control, 21(7), pp. 1092-1104, 2011.

[16] E. Harinath, L.T. Biegler, and G.A. Dumont. Control and optimization strategies for thermo-mechanical pulping processes: Nonlinear model predictive control. J. Process Control, 21(4), pp. 519-528, 2011.

[17] M. Chebre, Y. Creff, N. Petit Feedback control and optimization for the production of commercial fuels by blending. J. Process Control, 20(4), pp. 441-451, 2010.

[18] C.J. Muller, I.K. Craig, and N.L. Ricker. Modeling, validation, and control of an industrial fuel gas blending system. J. Process Control, 21(6), pp. 852-860, 2011.

[19] R.P. Dreyer and G.D. Kotze. Methods, systems, and articles for controlling a fluid blending system. International Patent Application No. PCT/US2004/013335, 2004.

[20] M. Misra, V. M. Marrero, K. S. Brooks, and H. de Beer. Energy Optimization through Non-Linear Modeling and Control of Fuel Gas Process at Hovensa. AIChE Annual Meeting, Paper 577s, 2008. 